\title{
Prevalence of Epidermal Growth Factor Receptor Exon 20 Insertion Mutations in Non-small-Cell Lung Cancer in Europe: A Pragmatic Literature Review and Meta-analysis
}

\author{
Suzy Van Sanden ${ }^{1}$ (1) $\cdot$ Molly Murton ${ }^{2} \cdot$ Anna Bobrowska $^{2} \cdot$ Nora Rahhali $^{3} \cdot$ Jan Sermon ${ }^{4} \cdot$ Bernardo Rodrigues $^{5}$. \\ Danielle Goff-Leggett ${ }^{2} \cdot$ Christos Chouaid $^{6} \cdot$ Martin Sebastian $^{7} \cdot$ Alastair Greystoke $^{8}$
}

Accepted: 3 February 2022 / Published online: 28 February 2022

(c) The Author(s) 2022

\begin{abstract}
Background Information on the epidemiology of uncommon EGFR mutations including exon 20 insertions amongst nonsmall-cell lung cancer (NSCLC) is lacking.

Objective The objective of this pragmatic literature review (PLR) and meta-analysis was to generate robust prevalence and incidence estimates based on ranges of exon 20 insertion mutations reported in the literature.

Materials and methods Searches of MEDLINE, Embase, congresses and reference lists for articles published from 2013 in key European countries of interest (Belgium, France, Germany, Italy, The Netherlands, Spain, Sweden, Switzerland, United Kingdom) were performed. Articles were reviewed against pre-specified criteria and their quality was appraised using a published checklist. Prevalence estimates were synthesised by random-effects meta-analyses.

Results Eighty unique studies of moderate-to-high quality were included in the PLR. The meta-analysed prevalence for EGFR mutations was $12.5 \%$ (95\% confidence interval [CI]: $11.0,14.1)$ in any stage NSCLC and $14.8 \%(12.8,17.1)$ in advanced/ metastatic NSCLC. The prevalence of exon 20 insertions was $0.7 \%(0.4,1.1)$ in any stage NSCLC and $6.1 \%(4.0,9.4)$ in any stage EGFR-positive NSCLC. Mutation status was primarily measured using direct sequencing or a combination of methods. One study reporting exon 20 insertions in advanced/metastatic disease was identified, which reported a prevalence of $0.5 \%$ in overall NSCLC and $4.0 \%$ in EGFR-positive NSCLC.

Conclusions EGFR exon 20 insertion mutations are rare in NSCLC. There is a high unmet need in patients with exon 20 insertions, including effective therapies. Prospective cohort studies are needed to better clinically characterise these patients.
\end{abstract}

\section{Introduction}

Joint primary authorship: Suzy Van Sanden and Molly Murton.

Suzy Van Sanden

svsande1@its.jnj.com

Janssen, Beerse, Belgium

2 Costello Medical, Cambridge, UK

3 Janssen Cilag, Île-de-France, France

4 Janssen Cilag, Beerse, Belgium

5 Janssen Cilag, Porto Salvo, Portugal

6 Department of Pneumology and Thoracic Oncology, Intermunicipal Hospital Centre of Créteil, Créteil, France

7 Department of Medicine, Hematology and Oncology, University of Frankfurt, Frankfurt, Germany

8 Sir Bobby Robson Clinical Trials Unit, Freeman Hospital, Newcastle upon Tyne, UK
Lung cancer is the leading cause of cancer-related deaths in men and the second cause in women, accounting for $18 \%$ of all cancer-related deaths worldwide $[1,2]$. In 2016, lung cancer accounted for approximately $20 \%$ of all cancer-related deaths and 5\% of all deaths in Europe [3]. Non-small-cell lung cancer (NSCLC) accounts for approximately $85 \%$ of all lung cancers and comprises three main histological subtypes. Adenocarcinoma is the most common, accounting for $\sim 60 \%$ of all NSCLC cases ( $~ 50 \%$ of all lung cancer), followed by squamous cell carcinoma $(\sim 25 \%$ of all NSCLC cases) [4].

NSCLC has several driver mutations, the majority of which occur in adenocarcinomas [5]. In adenocarcinoma, predominant alterations include mutations in Kirsten rat sarcoma virus $(K R A S)$, epidermal growth factor receptor $(E G F R)$ and anaplastic lymphoma kinase $(A L K)$ genes, 


\section{Key Points}

Data reporting on the epidemiology of EGFR exon 20 insertions in NSCLC are lacking.

A pragmatic literature review identified prevalence of EGFR exon 20 insertions.

Meta-analyses precisely quantified exact EGFR exon 20 insertion prevalence.

Results can be used to assess numbers of patients eligible for emerging therapies.

alongside ROS1, MEK1, HER 2 and RET genes. Other driver genes include FGFR1, PDGFRA, PTEN, MET, STK11, $B R A F$, PIK3CA, DDR2, AKT1 and NRF2 [5-8]. These mutations play important roles in the oncogenesis of NSCLC and are associated with numerous oncogenic effects, such as activating signalling proteins, leading to increased cell proliferation, metastasis and decreased apoptosis $[6,9]$.

EGFR is a transmembrane glycoprotein with an intracellular tyrosine kinase domain that contributes to the regulation of cell proliferation $[9,10]$. EGFR mutations have been reported to represent approximately $14 \%$ of all cases of NSCLC in European countries [11]. Mutations in the $E G F R$ gene can be categorised into four main types, based on the exon in which they occur. Common EGFR mutations are exon 19 deletions and exon 21 L858R point mutation, constituting approximately $85 \%$ of $E G F R$ mutations. The remaining $10-15 \%$ are made up of uncommon EGFR mutations, including exon 18 mutations and exon 20 insertion mutations, along with rarer exon 19 and 21 mutations, such as L861Q on exon 21 [12-14].

Exon 20 insertion mutations were among the earliest EGFR mutations to be identified and can be characterised as in-frame insertions or duplications between amino acid positions 762 and 774 of the EGFR protein (Fig. 1) [14, 15]. However, despite being one of the earliest identified mutations, data reporting on the epidemiology of exon 20 insertions are currently lacking $[14,16]$.

Uncertainty in epidemiological estimates is compounded by variations in testing methodologies for $E G F R$ mutations, which have varying degrees of sensitivity. For example, direct sequencing by polymerase chain reaction (PCR), which was the historical standard for EGFR mutation testing, has low sensitivity. It therefore only detects mutations when sufficient levels of mutant DNA are present [17]. Furthermore, PCR can only detect predetermined target sequences, meaning it has a low discovery power to detect novel genes and to quantify rare variants [18]. A number of alternative methods of mutation testing have been developed, such as next-generation sequencing (NGS), high-performance liquid chromatography (HPLC) and high-resolution melting analysis (HRMA). These methods have improved sensitivity and can detect more mutations using a relatively small amount of DNA [17]. They may also detect abnormalities not readily detected by previous PCR-based techniques, with a higher discovery power to detect all variants without prior sequence knowledge $[18,19]$.

Patients with activating EGFR mutations are typically treated with tyrosine kinase inhibitors (TKIs), which have positive results for NSCLC with common EGFR mutations [20]. However, therapy options for patients with rare $E G F R$ mutations have historically been lacking. Exon 20 insertion mutations are associated with de novo resistance to TKIs, and many TKIs, such as osimertinib and poziotinib, have been shown to have limited efficacy against exon 20 insertion mutations [21, 22]. The limited efficacy of potential treatments has contributed to the poorer prognosis reported for these patients compared with patients with other EGFR mutations [23]. A 2020 study reported a 75\% increased risk of death with exon 20 insertion mutations compared with common EGFR mutations, as well as $8 \%$ of patients achieving 5-year survival compared with $19 \%$ of patients with common EGFR mutations [24].

However, recently developed investigational therapies such as amivantamab, a monoclonal antibody, and mobocertinib, a small-molecule TKI, are expected to have specific activity against exon 20 insertion mutations. In 2021, amivantamab was granted approval by the US Food and Drug Administration (FDA) and European Medicines Agency (EMA) [25, 26]; mobocertinib was granted approval by the FDA based on positive clinical trial data [27]. There is a need for accurate estimates of the number of patients that would benefit from such emerging therapies.

A systematic literature review (SLR) and meta-analysis (with searches run in 2013) conducted by Zhang 2016 reported on the frequency of EGFR mutations, including exon 20 insertion mutations, in NSCLC. It found a pooled frequency of $1.7 \%(1.4,2.0)$ for exon 20 insertion mutations in any stage NSCLC in Europe [11]. However, this estimate may now be outdated. More recently, other sources have reported a wide range in estimates, for example an SLR conducted by Burnett 2021 reported a frequency of exon 20 insertion mutations in Europe which ranged from 0.3 to $1.3 \%$ in all NSCLC cases, and 4-12\% in EGFR-positive NSCLC [23]. As a meta-analysis was not performed, the true prevalence cannot be ascertained.

Lung cancer accounts for approximately $5 \%$ of all deaths in Europe, ranging from $4 \%$ in Sweden to $7 \%$ in the Netherlands. The objective of this pragmatic literature review (PLR) was to identify current estimates of the incidence and prevalence of $E G F R$ exon 20 insertion mutations in NSCLC in nine key European countries (Belgium, France, Germany, Italy, the Netherlands, Spain, Sweden, Switzerland and the 


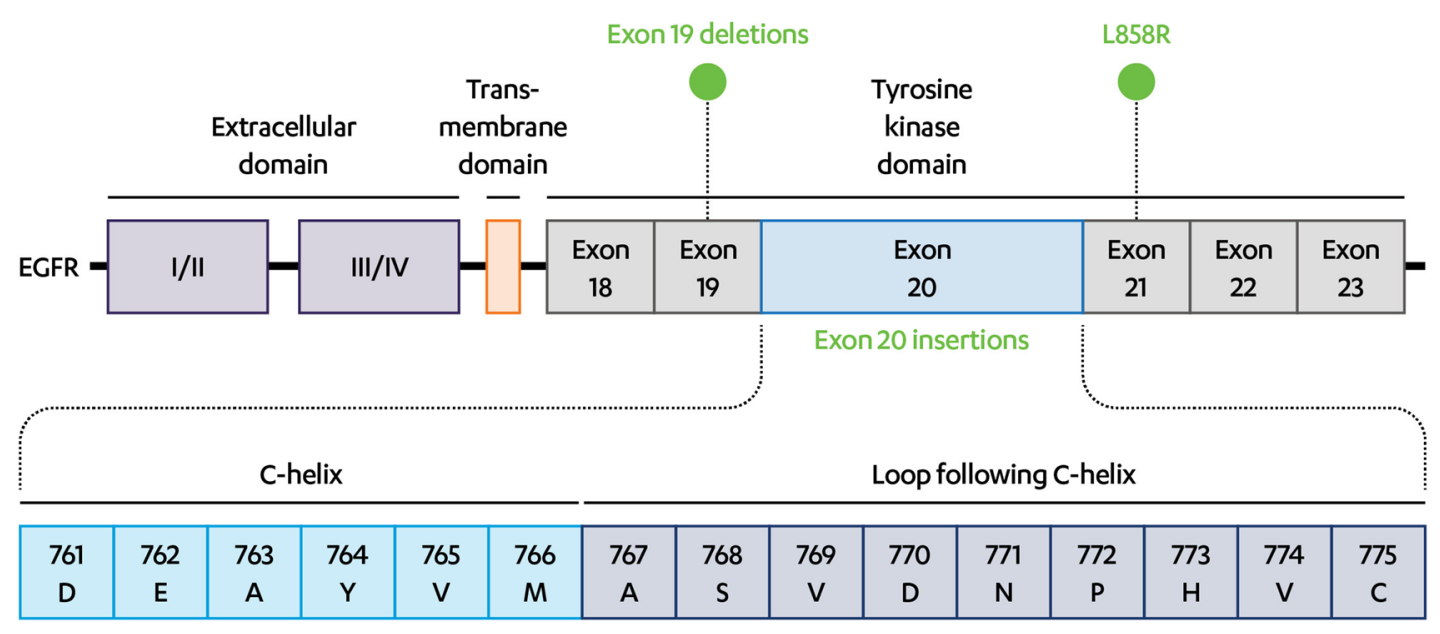

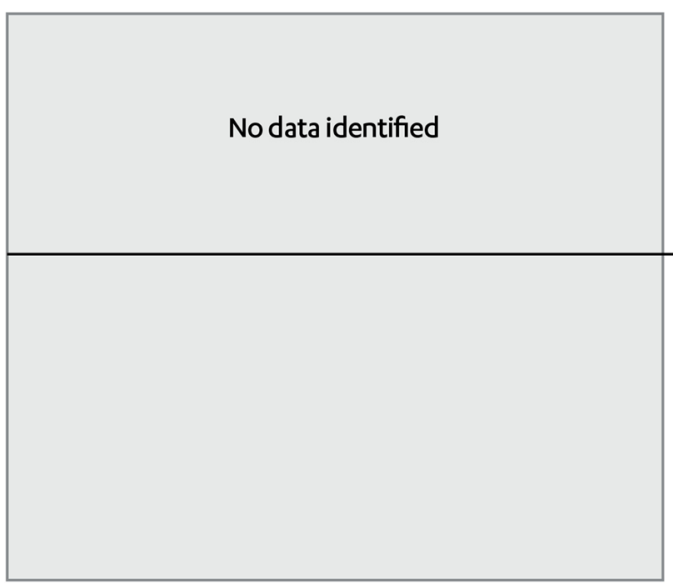

Fig. 1 EGFR exon 20 insertion mutations in NSCLC [14]. Prevalence

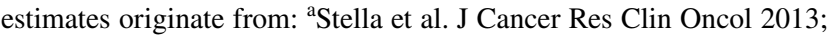
139:1327-1335; 'bocatelli-Sanchez et al. Lung 2013;191:491-499; ${ }^{c}$ Kerner et al. PLoS One 2013;8:e70346. Exon 19 deletions and L858R exon 21 mutations are considered common EGFR mutations.

UK). These countries were selected as current European prevalence estimates are outdated, lack granularity and are wide ranging $[11,23]$.

The identified ranges for prevalence estimates from the PLR were wide. As such, the true prevalence of EGFR-positive and exon 20 insertion positive NSCLC was unclear. Therefore, a subsequent meta-analysis was conducted with the objective of establishing more accurate estimates of the epidemiology of EGFR-positive NSCLC in these countries, including exon 20 insertions.

\section{Materials and Methods}

The PLR was performed in accordance with a pre-specified protocol and reported in accordance with the Preferred Reporting Items for Systematic Reviews and Meta-Analyses (PRISMA) statement. The review protocol was not registered.
Figure source: Vyse S. et al Signal Transduct Target Ther. 2019;4:110. Adapted based on evidence identified by the pragmatic literature review. EGFR epidermal growth factor receptor, NSCLC non-smallcell lung cancer

\subsection{Identification of Studies}

The SLR conducted by Zhang 2016 collected data from 456 studies worldwide, published up to June 2013 [11]. In order to avoid the duplication of work and to capture only the most recent evidence using modern testing technologies, the searches for this PLR were limited to 2013 or later. The Zhang 2016 SLR was judged to be of high quality according to the AMSTAR 2 critical appraisal tool, and therefore a reliable source of reference data [28].

A comprehensive approach was taken to evidence identification. Electronic database searches were conducted in MEDLINE and Embase simultaneously via Ovid on 5 May 2020. The complete search strategy is presented in the Online Supplementary Material (OSM) Table S1. Additionally, conference proceedings from the last three years (2018 to 2020) were searched for the American Association for Cancer Research (AACR), the American Society of Clinical Oncology (ASCO), the European Society for Medical 
Oncology (ESMO), the International Association for the Study of Lung Cancer (IASLC) European Conference on Lung Cancer and ESMO Lung Cancer Annual Congress. Bibliographies of published SLRs and/or (network) metaanalyses identified during the searches were also reviewed to identify additional relevant publications.

\subsection{Selection of Studies}

Each abstract and full text was assessed for inclusion by a single reviewer using pre-defined eligibility criteria, based on the population(s), intervention(s), comparator(s) and outcome(s) (PICO) framework (OSM Table S2). Studies were included if they reported the prevalence or incidence of mutations in the $E G F R$ gene in patients in the following European countries: Belgium, France, Germany, Italy, the Netherlands, Spain, Sweden, Switzerland, UK. To avoid selection bias, only observational studies were included. A second independent reviewer checked all included and $10 \%$ of the excluded articles.

\subsection{Data Extraction and Reporting of Results}

Key information from each included study, including study characteristics, patient characteristics and epidemiological outcomes, was extracted into a pre-specified data extraction grid by a single individual. A second individual independently verified $10 \%$ of the extracted information (with a particular focus on the quantitative data) to ensure its accuracy. There was a low discrepancy rate in the verified data $(1.06 \%)$; therefore, there were low concerns about the quality of the unverified extractions.

Where not reported in the publication, simple calculations were performed to generate prevalence estimates, if possible based on the available data. Results for the same outcome across different studies, for example EGFR mutation frequency, were used to inform a range from lowest reported value to highest reported value. The more common T790M mutation was not included within estimates of exon 20 insertion prevalence.

\subsection{Quality Assessment}

The quality of all included studies was assessed using the Joanna Briggs Institute (JBI) Critical Appraisal tools Checklist for Prevalence Studies [29]. The quality assessment was completed by one individual and $10 \%$ of the information was verified by a second independent reviewer.

\subsection{Meta-analysis}

Meta-analyses were conducted to synthesise the prevalence estimates identified by the PLR. Specifically, metaanalyses were performed for the prevalence of overall
$E G F R$ mutations in any stage NSCLC, EGFR mutations in advanced/metastatic NSCLC, EGFR mutations in patient subgroups (men and women, smokers and non-smokers, adenocarcinoma and non-adenocarcinoma) and the frequency of exon 20 insertion mutations in NSCLC and in EGFRpositive NSCLC. The prevalence reported by each study was analysed using logistic regression with a logit transformation. Prevalence reported by individual studies was used as the input for the models, along with their calculated exact Copper-Pearson confidence intervals (CIs). The $I^{2}$ statistic was used to assess whether fixed-effect or random-effect models were appropriate. A maximum likelihood estimator was used to estimate the between-study variation, $\tau^{2}$, and all meta-analyses were run using statistical software, R, [30] with the function metaprop from the meta package [31].

\section{Results}

\subsection{Study Selection}

A total of 1230 records were retrieved by the electronic database searches. After de-duplication of results, 1217 unique records were reviewed (Fig. 2). After title and abstract review, 163 records were selected for full-text review. Of these, 90 were found to fulfil the eligibility criteria for inclusion in the review. Supplementary searches of congresses and SLR bibliographies yielded three additional records that fulfilled the eligibility criteria. In total, 93 publications reporting 80 unique studies were included in the review.

Forty studies reported the prevalence of overall EGFR mutations in any stage NSCLC and 30 studies reported the prevalence of overall EGFR mutations in advanced/metastatic NSCLC. Nine studies reported on exon 20 insertion mutations in any-stage NSCLC and one study reported on exon 20 insertion mutations specifically in advanced/metastatic NSCLC (Table 1).

\subsection{Study Characteristics}

All included studies reported on the prevalence of mutations. No evidence was identified for incidence. Across all studies, data were collected between 2008 and 2017. The nine studies that reported the frequency of exon 20 insertions in any-stage NSCLC were located in France $(n=4)$ [32-35], Italy $(n=1)$ [36], UK $(n=2)[37,38]$, the Netherlands $(n=$ 1) [39] and Sweden $(n=1)$ [40]. The one study reporting the frequency of exon 20 insertions in advanced or metastatic NSCLC was conducted in Spain (Table 1) [41].

Study population size ranged widely, from 134 participants in a study performed in Italy to 10,117 participants in the French ERMETIC-IFCT study [32, 36]. The nine studies reporting on exon 20 insertions in any-stage NSCLC included a 


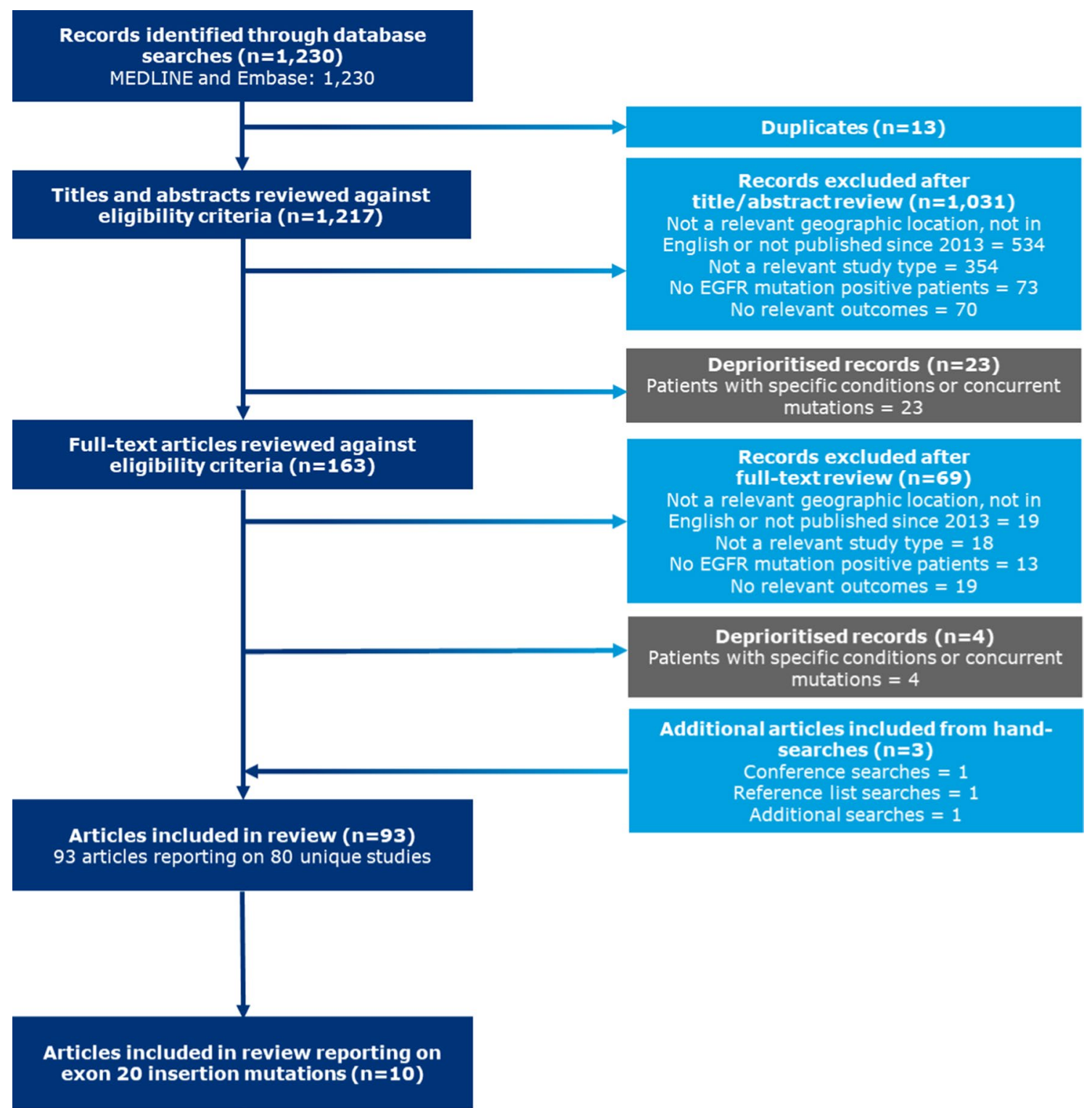

Fig. 2 PRISMA diagram. Due to the large volume of evidence identified throughout the review, a prioritisation strategy was implemented in order to synthesise evidence and draw conclusions of the highest possible utility. The deprioritised evidence included: studies with specific patient populations, such as patients with specific conditions (e.g. HIV, Li-Fraumeni syndrome) or patients selected based on having specific mutations (e.g. METex14, ALK translocations,

pooled total of 32,236 participants. The one study that reported on exon 20 insertion mutations specifically in advanced/metastatic NSCLC included 184 participants [41]. Although the majority of studies included patients with all stages of NSCLC, it was noted that a high proportion of these patients were stage III or IV NSCLC (ranging from 28 to $99 \%$ ) (Table 1).

Direct sequencing by PCR was used to identify and measure $E G F R$ mutations in the majority of studies. However, most studies also reported using more than one method,
MAP2K1, HER2, BRAF). ALK anaplastic lymphoma kinase, BRAF v-raf murine sarcoma viral oncogene homolog B1, EGFR epidermal growth factor receptor, HER2 human epidermal growth factor 2, HIV human immunodeficiency virus, MAP2K1 mitogen-activated protein kinase 1, METex14 mesenchymal-epithelial transition gene exon 14, PRISMA Preferred Reporting Items for Systematic Reviews and Meta-Analyses

such as fragment analysis and NGS (Table 1). PCR methods included PCR-high resolution melt (HRM) and allelespecific PCR, such as the Therascreen EGFR Rotor-Gene Q (RGQ) PCR kits.

Of the ten included studies, eight were reporting results in TKI-naïve patients. Of the two studies that included patients who had been exposed to prior therapies, the ERMETICIFCT study publication specified these therapies included erlotinib and gefitinib [32, 35]. 


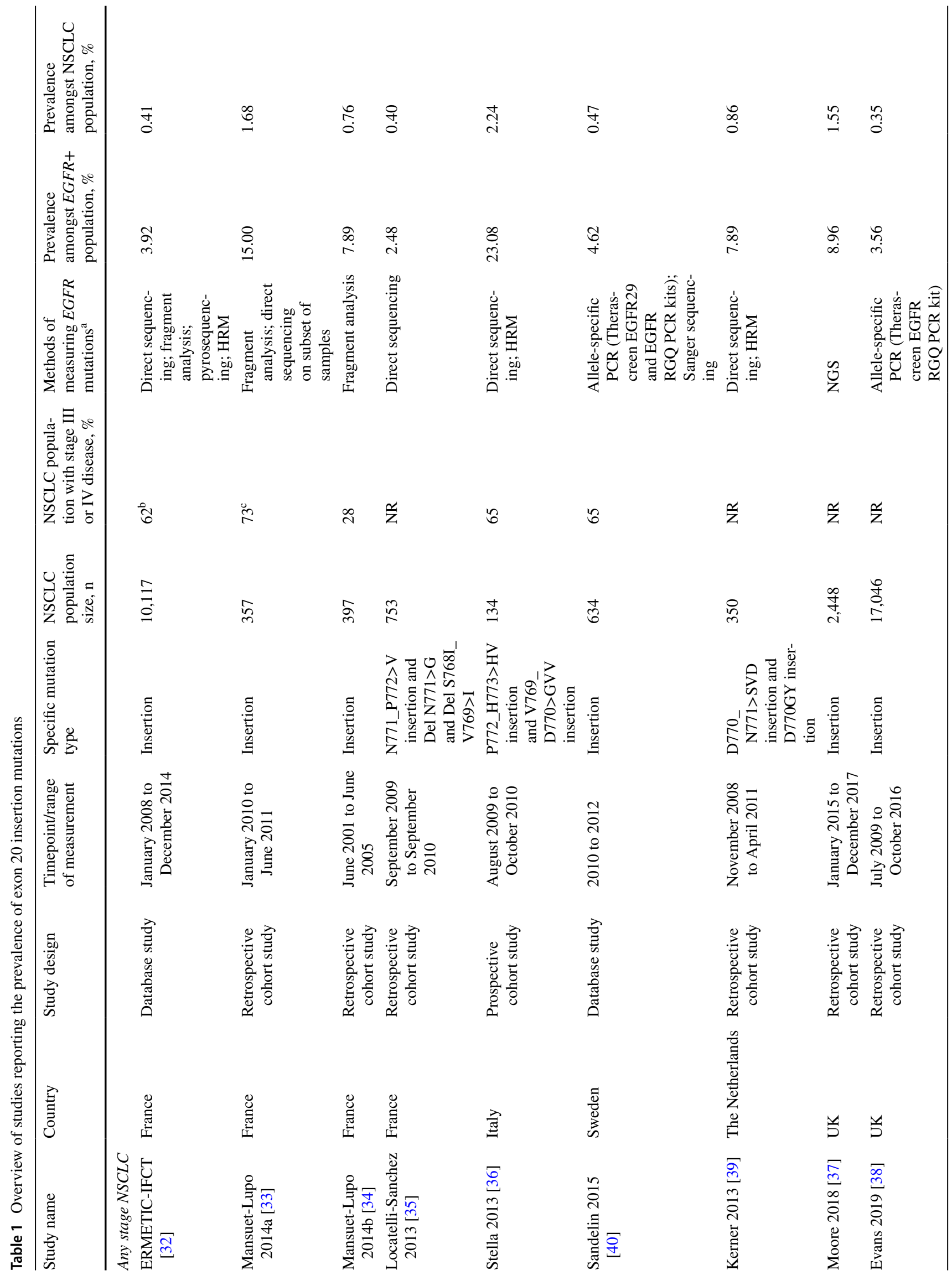




\subsection{Study Quality}

Overall, the risk of bias of the ten included studies reporting exon 20 insertion mutations was deemed to be low-to-moderate, based on the questions in the adapted JBI checklist [29]. A summary of the quality for each question domain and each study is presented in OSM Table S3.

Of the included studies, 7/10 had a primary aim of identifying mutation prevalence or frequency [32-34, 38-41]. The remaining three studies did not have the primary aim of measuring mutation frequency [35-37], rather data were collected as part of routine clinical work.

The majority (9/10) of studies performed well in the domains of sufficient coverage of the identified sample and valid methods for testing mutational status in that most studies included all patients for whom mutational status was available and standard methods of testing including direct sequencing or fragment analysis were typically used [32-38, 40, 41].

\subsection{Estimated Prevalence of Overall EGFR Mutations and Exon 20 Insertion Mutations from the Pragmatic Literature Review}

The frequency of overall EGFR mutations in any stage NSCLC ranged from $4.0-28.9 \%$, and $5.0-37.5 \%$ in advanced/metastatic NSCLC (Table 2; Fig. 3). The overall frequency of exon 20 insertion mutations in any stage NSCLC ranged from $0.3-2.2 \%$ amongst a general NSCLC population and 2.5-23.1\% within EGFR-positive NSCLC, based on nine studies (Table 2; Fig. 3).

The lowest reported frequency of exon 20 insertions within an overall NSCLC population (0.4\%) was in a study conducted in the UK [38]. The lowest value within an EGFRpositive NSCLC population (2.5\%) was reported in a study conducted in France [38]. The highest reported frequency of exon 20 insertions within both an overall NSCLC population and an EGFR-positive NSCLC population was in a study conducted in Italy (2.2\% and $23.1 \%$, respectively) [36]. This Italian study had the smallest sample size of all studies reporting on exon 20 insertions, at 134 patients. It reported rates of three separate mutations (N771-P772 insV, del N771 insG and del S768I-V769 insI), which were summed to give a total value under the assumption that patients would not have multiple exon 20 insertion mutations.

The two largest studies (ERMETIC-IFCT in France, $n$ $=10,117$ and Evans 2019 in the UK, $n=17,046$ ), both reported similar frequencies of exon 20 insertions [32, 38]. ERMETIC-IFCT found 0.4\% and Evans 2019 found $0.4 \%$ of all patients with NSCLC had exon 20 insertions, and exon 20 insertions constituted $3.9 \%$ and $3.6 \%$ of EGFRpositive NSCLC, respectively [32, 38]. Evans 2019 used allele-specific PCR to measure EGFR mutations, and in 
Table 2 Frequency of EGFR mutations including exon 20 insertions in any stage and advanced/metastatic NSCLC

\begin{tabular}{|c|c|c|c|c|c|c|}
\hline & \multicolumn{3}{|c|}{ Prevalence in any stage NSCLC, $\%$} & \multicolumn{3}{|c|}{ Prevalence in advanced/metastatic NSCLC, $\%$} \\
\hline & \multirow[t]{2}{*}{ Any $E G F R$} & \multicolumn{2}{|c|}{ Exon 20 insertions } & \multirow[t]{2}{*}{ Any $E G F R$} & \multicolumn{2}{|c|}{ Exon 20 insertions } \\
\hline & & In NSCLC & In $E G F R+$ NSCLC & & In NSCLC & In $E G F R+$ NSCLC \\
\hline Any country & $\begin{array}{l}4.0-28.9 \\
40 \text { studies } \\
n=180,104\end{array}$ & $\begin{array}{l}0.3-2.2 \\
9 \text { studies } \\
n=32,236\end{array}$ & $\begin{array}{l}2.5-23.1 \\
9 \text { studies } \\
n=32,236\end{array}$ & $\begin{array}{l}5.0-37.5 \\
30 \text { studies } \\
n=17,103\end{array}$ & $\begin{array}{l}0.5 \\
1 \text { study } \\
n=184\end{array}$ & $\begin{array}{l}4.0 \\
1 \text { study } \\
n=184\end{array}$ \\
\hline The Netherlands & $\begin{array}{l}10.9 \\
1 \text { study } \\
n=350\end{array}$ & $\begin{array}{l}0.9 \\
1 \text { study } \\
n=350\end{array}$ & $\begin{array}{l}7.9 \\
1 \text { study } \\
n=350\end{array}$ & $\begin{array}{l}8.9 \\
1 \text { study } \\
n=325\end{array}$ & NR & NR \\
\hline Sweden & $\begin{array}{l}10.3 \\
1 \text { study } \\
n=634\end{array}$ & $\begin{array}{l}0.5 \\
1 \text { study } \\
n=634\end{array}$ & $\begin{array}{l}4.6 \\
1 \text { study } \\
n=634\end{array}$ & $\begin{array}{l}21.2 \\
1 \text { study } \\
n=831\end{array}$ & NR & NR \\
\hline Switzerland & $\begin{array}{l}19.2 \\
1 \text { study } \\
n=469\end{array}$ & NR & NR & $\begin{array}{l}11.4-37.5 \\
2 \text { studies } \\
n=463\end{array}$ & NR & NR \\
\hline UK & $\begin{array}{l}4.0-17.3 \\
5 \text { studies } \\
n=19,745\end{array}$ & $\begin{array}{l}0.3-1.6 \\
2 \text { studies } \\
n=19,494\end{array}$ & $\begin{array}{l}3.6-9.0 \\
2 \text { studies } \\
n=19,494\end{array}$ & $\begin{array}{l}10.1 \\
1 \text { study } \\
n=701\end{array}$ & NR & NR \\
\hline Spain & $\begin{array}{l}5.9-21.3 \\
7 \text { studies } \\
n=4561\end{array}$ & NR & NR & $\begin{array}{l}9.9-22.2 \\
9 \text { studies } \\
n=4697\end{array}$ & $\begin{array}{l}0.5 \\
1 \text { study } \\
n=184\end{array}$ & $\begin{array}{l}4.0 \\
1 \text { study } \\
n=184\end{array}$ \\
\hline Italy & $\begin{array}{l}9.7-27.5 \\
7 \text { studies } \\
n=3406\end{array}$ & $\begin{array}{l}2.2 \\
1 \text { study } \\
n=134\end{array}$ & $\begin{array}{l}23.1 \\
1 \text { study } \\
n=134\end{array}$ & $\begin{array}{l}5.0-24.8 \\
7 \text { studies } \\
n=1725\end{array}$ & NR & NR \\
\hline France & $\begin{array}{l}5.0-16.1 \\
8 \text { studies } \\
n=146,633\end{array}$ & $\begin{array}{l}0.4-1.7 \\
4 \text { studies } \\
n=11,624\end{array}$ & $\begin{array}{l}2.5-15.0 \\
4 \text { studies } \\
n=11,624\end{array}$ & $\begin{array}{l}10.3-24.5 \\
7 \text { studies } \\
n=4011\end{array}$ & NR & NR \\
\hline Germany & $\begin{array}{l}4.9-28.9 \\
10 \text { studies } \\
n=4306\end{array}$ & NR & NR & $\begin{array}{l}10.3-17.0 \\
2 \text { studies } \\
n=4350\end{array}$ & NR & NR \\
\hline Belgium & NR & NR & NR & NR & NR & NR \\
\hline
\end{tabular}

EGFR epidermal growth factor receptor, NR not reported, NSCLC non-small-cell lung cancer

ERMETIC-IFCT several different methods including direct sequencing, fragment analysis, pyrosequencing and PCRHRM were used across different study centres.

The GGCP 048-10 study, which was the only study to report on the frequency of exon 20 insertions in advanced/ metastatic NSCLC, found a frequency of one exon 20 insertion amongst 184 patients with NSCLC, or among 25 patients with EGFR-positive NSCLC ( $0.5 \%$ in NSCLC; $4.0 \%$ in EGFR-positive NSCLC) [41].

Seven studies reported on "Insertions" in exon 20 [32-34, $37,38,40,41]$, while three studies reported on specific insertions [35, 36, 39]. These included N771_P772>V $(0.1 \%$ in overall NSCLC; $0.8 \%$ in EGFR-positive NSCLC) [35], $\mathrm{P} 772 \_\mathrm{H} 7773>\mathrm{HV}$ (1.5\% in overall adenocarcinoma; $15.6 \%$ in EGFR-positive adenocarcinoma) [36], V769_D770>GVV (0.75\% in overall adenocarcinoma; $7.7 \%$ in EGFR-positive adenocarcinoma) [36], D770_N771 >SVD $(0.3 \%$ in overall NSCLC; $2.6 \%$ in EGFR-positive NSCLC) [39], D770G $>Y$ (0.6\% in overall NSCLC; $5.3 \%$ in EGFR-positive NSCLC) [39], del N771 $>\mathrm{G}(0.1 \%$ in overall NSCLC; $0.8 \%$ in
EGFR-positive NSCLC) [35] and del S768I_V769>I (0.1\% in overall NSCLC; $0.8 \%$ in EGFR-positive NSCLC) [35] (Fig. 1).

For exon 20 insertions in any stage NSCLC, the earliest year of data collection was 2001 and the latest year was 2015. For advanced/metastatic NSCLC, the GGCP 048-10 study collected data in 2011 (Fig. 4).

The most common method for measuring mutation status was direct sequencing using PCR or a combination of methods. Only one study, conducted in the UK (Moore 2018), used NGS to measure exon 20 insertion mutation (Fig. 4) [37]. This study had the most recent data collection (starting in 2015) and reported a prevalence estimate for exon 20 insertions of $1.6 \%$ in overall NSCLC and $9.0 \%$ in an EGFRpositive population. These values were higher than the estimates from the majority of studies, including the two largest studies (Evans 2019 and ERMETIC-IFCT) (Fig. 4) [32, 38].

Complex mutations or co-mutations (the occurrence of multiple different mutations in a single tumour) involving exon 20 insertion mutations were reported in five of the ten 


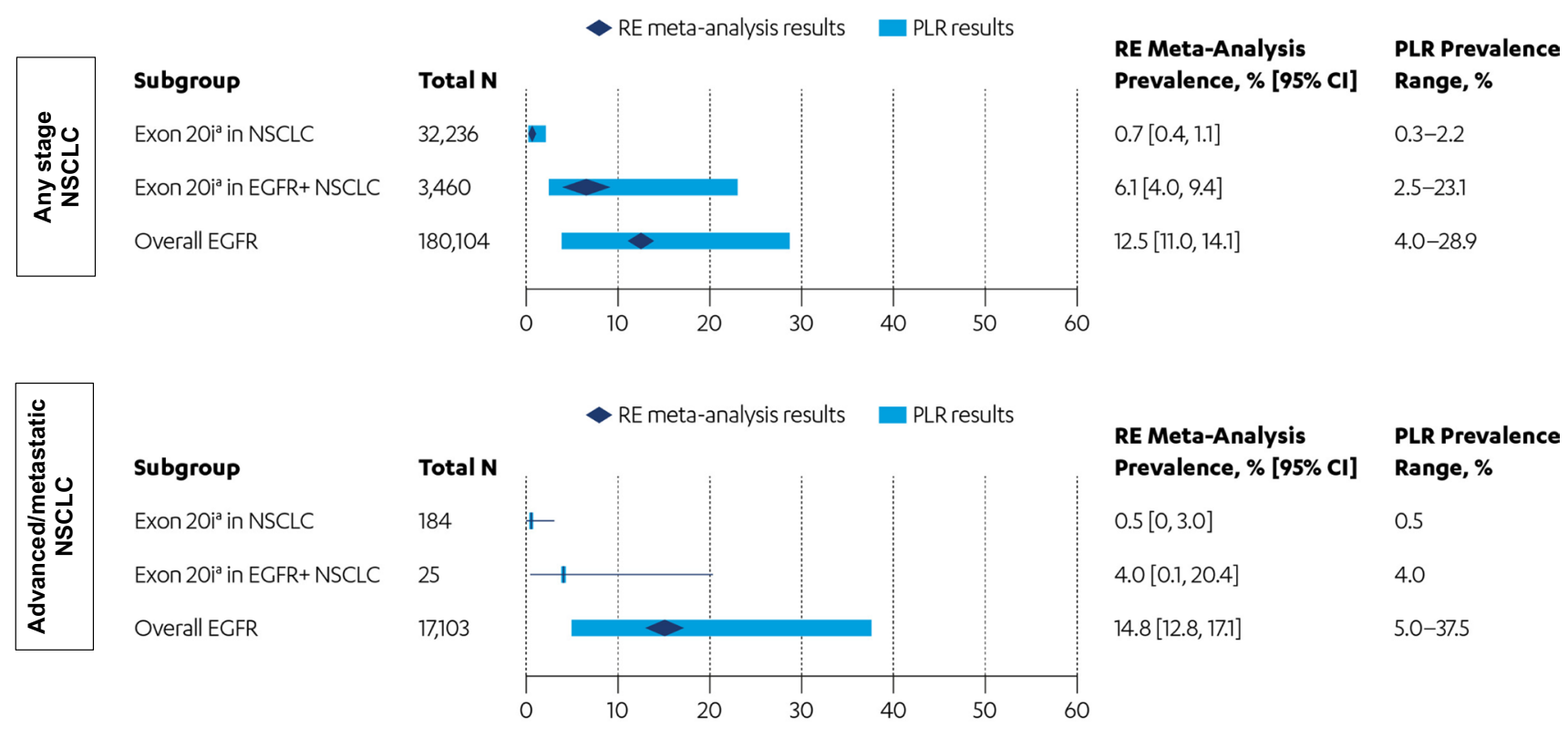

Fig. 3 Estimated frequency from the meta-analysis and PLR of exon 20 insertion mutations in any stage and advanced/metastatic NSCLC. ${ }^{a}$ Does not include T790M. The figures present a summary of the pooled results from the random effects meta-analyses overlayed with the range of results from the PLR. Full forest plots for the individual meta-analyses are presented in the supplementary materials. The out- come for the exon 20 insertion mutation frequency in advanced/metastatic NSCLC is based on findings of the one study. $C I$ confidence interval, EGFR epidermal growth factor receptor, exon $20 \mathrm{i}$ exon 20 insertion mutations, NSCLC non-small-cell lung cancer, PLR pragmatic literature review, $R E$ random effects

same number of publications as identified in the PLR were included in the meta-analysis.

The overall analysed prevalence for EGFR mutations was $12.5 \%$ (95\% CI: 11.0, 14.1) (PLR range: 4.0-28.9\%) in any stage NSCLC and $14.8 \%(12.8,17.1)$ (PLR range: 5.0-37.5\%) specifically in advanced/metastatic NSCLC (Fig. 3; OSM Fig. S1). The overall analysed prevalence of exon 20 insertions in any-stage NSCLC was $0.7 \%(0.4,1.1)$ (PLR range: $0.3-2.2 \%)$, and $6.1 \%(4.0,9.4)$ (PLR range: 2.5-23.1\%) in EGFR-positive NSCLC (Fig. 3; OSM Fig. $\mathrm{S} 1$ ). The CIs generated from the meta-analysis are considerably narrower than the ranges across studies identified in the PLR, and therefore provide a more precise single point estimate than the studies identified in the PLR.

Only one study reported the frequency of exon 20 insertions specifically in advanced/metastatic NSCLC, therefore a meta-analysis of these results was not conducted. This study showed a prevalence of $0.5 \%$ (calculated $95 \%$ CI: $0,3.0$ ) in overall NSCLC and 4.0 (calculated 95\% CI: 0.1, 20.4) in EGFR-positive NSCLC (Fig. 3; OSM Fig. S1) [41].

None of the studies reporting on exon 20 insertion mutations presented results separately for specific patient subgroups, for example stratified by sex, smoking status or NSCLC histology. However, these data were reported separately in studies investigating overall EGFR mutation frequency, with higher $E G F R$ frequency in adenocarcinoma (12.7\%) versus non-adenocarcinoma (1.8\%), women $(20.1 \%)$ 


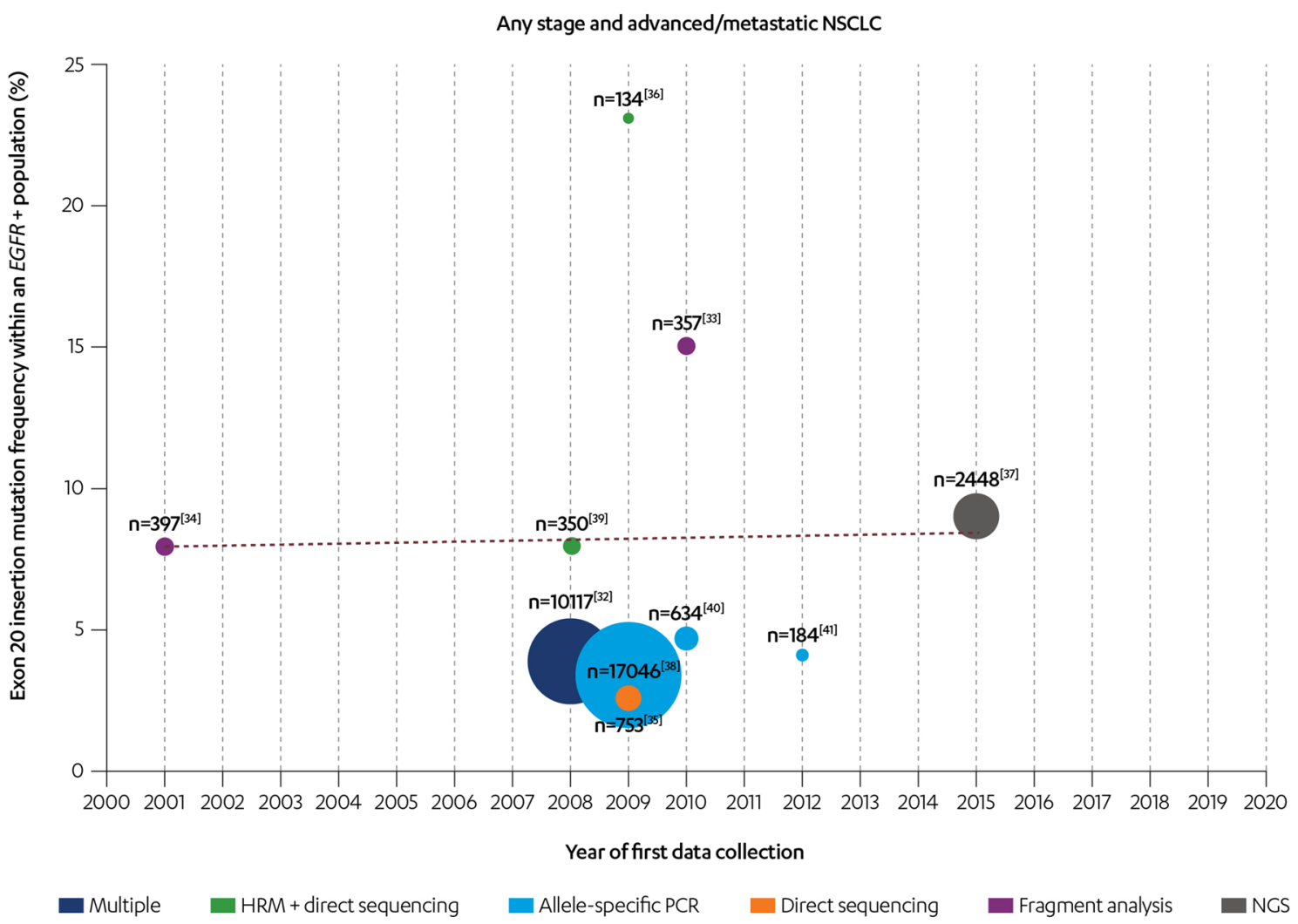

Fig. 4 Frequency of exon 20 insertion mutations in any stage NSCLC and advanced/metastatic NSCLC per year and method of data collection. 'Multiple' = direct sequencing, fragment analysis, pyrosequencing and HRM. Bubble size represents the number of patients

versus men (7.2\%) and non-smokers (43.1\%) versus smokers (4.8\%) with NSCLC (OSM Fig. S2).

\section{Discussion}

This PLR and meta-analysis identified substantial moderateto high-quality evidence on the frequency of EGFR mutations in NSCLC. The overall prevalence of EGFR mutations identified by the PLR ranged from 4.0 to $28.9 \%$ in any stage NSCLC, and 5.0-37.5\% in advanced/metastatic NSCLC. The single-point estimates and $95 \%$ CIs obtained by the meta-analyses for EGFR mutations were $12.5 \%$ (11.0, 14.1) in any-stage NSCLC and $14.8 \%(12.8,17.1)$ in advanced/ metastatic NSCLC. These values align well with previously reported estimates in the literature [11], increasing confidence that the exon 20 insertion results identified in this PLR and meta-analysis are robust.

The prevalence of exon 20 insertion mutations identified by this PLR in any-stage NSCLC ranged from 0.3 to $2.2 \%$ in overall NSCLC and 2.5-23.1\% in EGFR-positive NSCLC. In advanced/metastatic NSCLC, the prevalence of exon 20 with NSCLC in each study. Numbers in square brackets represent the article citation. EGFR epidermal growth factor receptor, HRM highresolution melt, NGS next-generation sequencing, NSCLC non-smallcell lung cancer

insertion mutations was $0.5 \%$ in overall NSCLC and $4.0 \%$ in EGFR-positive NSCLC. The single-point estimates and 95\% CIs obtained by the meta-analyses for exon 20 insertions in any stage NSCLC were $0.7 \%(0.4,1.1)$ for overall NSCLC and $6.1 \%(4.0,9.4)$ in EGFR-positive NSCLC. In advanced/metastatic NSCLC, prevalence was $0.5 \%(0,3.0)$ in overall NSCLC and $4.0 \%(0.1,20.4)$ in EGFR-positive NSCLC. The meta-analysis results provide more exact exon 20 insertion mutation prevalence data, adding value to the broad prevalence ranges identified by the PLR.

The majority of studies reporting on exon 20 insertion mutations were conducted in France (4/10), and these studies contained the largest sample sizes [32-35]. The one study that used NGS reported a higher prevalence than the majority of studies that used other testing methodologies,[37] including the two largest studies, indicating that NGS may be a more sensitive test to identify these mutations. The majority of studies used direct sequencing, either alone or in combination with other methods of mutation detection. For these studies, prevalence rates ranged from $2.5 \%$ to $23.1 \%$, suggesting no clear consistency in results between studies using the same method of mutation detection. Additionally, 
no clear trend was identified between method of mutation assessment or year of data collection and EGFR mutation frequency in any stage or advanced/metastatic NSCLC, meaning that conclusions about their contribution to heterogeneity in results cannot be drawn.

The SLR and meta-analysis conducted by Zhang 2016 (the reference source for this PLR) and another recentlyconducted SLR, Burnett 2021, can be used to contextualise this review's findings [11, 23]. Zhang 2016 included 456 studies (62 from Europe). The EGFR mutation frequency in Europe was $14.1 \%$ (95\% CI 12.7, 15.5\%). This was consistent with the results of this meta-analysis for EGFR mutation frequency $(12.5 \%$ [11.0, 14.1]). Zhang 2016 did not report prevalence values for specific countries but did report frequency for stage III and stage IV NSCLC at $33.8 \%$ (29.8, $7.8)$ and $37.5 \%(33.2,41.7)$, respectively. These are considerably higher than the estimated prevalence for advanced/ metastatic disease from the current meta-analysis (14.8\% $[12.8,17.1])$. Additionally, the frequency of exon 20 insertion mutations in any stage NSCLC was reported at $1.7 \%$ $(1.4,2.0)$ by Zhang 2016 . This is also higher than the estimated prevalence from this meta-analysis $(0.7 \%[0.4,1.1]$ in any-stage NSCLC and $0.5 \%[0,3.0]$ in advanced/metastatic NSCLC). This difference may be explained by the fact that Zhang 2016 included locations outside of Europe (including Asia and North and South America) for the exon 20 insertion estimate. For context, EGFR mutation prevalence in any-stage NSCLC was substantially higher in Asia and America (38.4\% [36.5, 40.3] and 24.4\% [22.1, 26.8]) [11]. Additionally, the race of patient populations may contribute to the difference. In all of the studies included in this PLR that reported ethnicity, $\geq 90 \%$ patients were White. In the Zhang 2016 SLR, rates of EGFR mutation were significantly higher for subgroups of Asian patients (38.8\%) compared with Caucasian (17.4\%) or African-American (17.2\%) patients [11]. This explanation is supported by other studies that have specifically investigated exon 20 insertions in other regions, with frequencies as high as $4.0 \%$ for Asia Pacific, $2.6 \%$ for the USA and 2.1\% for Latin America [23]. This re-emphasizes the need for prevalence estimates specifically for European regions as epidemiological conclusions cannot be generalised across geographical regions.

Burnett 2021 included 78 studies reporting on the frequency of exon 20 insertion mutations (13 from Europe), which in Europe ranged from 0.3 to $1.3 \%$ of all NSCLC cases, and $4-12 \%$ in EGFR-positive NSCLC [23]. This aligns with the range identified in this PLR $(0.3-2.2 \%$ amongst a general NSCLC population and $2.5-23.1 \%$ within an EGFR-positive NSCLC population), particularly when excluding the small Italian study that reported values at the upper limit of the range or studies that did not have a primary aim of identifying mutation prevalence or frequency. Burnett 2021 did not conduct a meta-analysis, therefore a more precise prevalence estimate was not generated and comparisons cannot be made with the results from the metaanalyses performed in this work [23].

A key strength of this review includes the identification and synthesis of recent, up-to-date evidence for all EGFR mutations, with a particular focus on exon 20 insertions. Furthermore, the meta-analysis provides the added value of synthesising the available evidence from the scientific literature to provide accurate point estimates of prevalence. Although only estimates for exon 20 insertion mutations are reported here, this PLR additionally identified data on other mutation types, including exon 18 mutations, which could contribute to valuable future research. The review was targeted to nine European countries that were of particular interest as previously reported estimates of prevalence in these countries were largely outdated and wide-ranging. Results from this PLR and meta-analysis provide current insights and add to the epidemiological understanding of exon 20 insertion mutations in these countries.

However, there are some limitations to this work. Regarding the review methodology, a single-reviewer approach was used during the study selection and data extraction. As such, it is possible that not all relevant articles were captured, and errors may have been introduced during data extraction. However, it is likely that the articles with high relevance to the review question will have been identified and a second individual independently verified $10 \%$ of the extracted information, where there was a low discrepancy rate in the verified data (1.06\%). As a result, there are low concerns about missing studies and the quality of the unverified extractions.

There were also limitations in the evidence base. Firstly, only prevalence data were identified, with no reported evidence for the incidence of exon 20 insertion mutations, and data specifically in advanced/metastatic populations of NSCLC were only reported by one study (with other studies including varied proportions of patients with stage I-IV disease). There is therefore higher uncertainty in the results and conclusions for this key population. Furthermore, heterogeneity between different studies, including study design and patient baseline characteristics may mask true differences between different markets or populations. Data reporting on the epidemiology of exon 20 insertion mutations were not reported for subgroups, such as sex, histological subtype and smoking status, so no conclusions can be drawn on how these factors may influence mutation frequency. Additionally, the occurrence of co-mutations or complex mutations and variation in testing methodologies may confound the measured frequency of exon 20 insertion mutations and cause their occurrence to be underestimated. More sensitive mutation testing methods may be required to accurately detect and distinguish between uncommon EGFR mutations to allow for more accurate prevalence estimates. 
Lastly, with the PLR's searches conducted in May 2020, more recently published evidence has not been accounted for. However, a targeted PubMed search in January 2022 identified two key studies reporting exon 20 insertions in European populations since May 2020. One was a large realworld cohort study from France that included 9,435 patients with non-squamous NSCLC [42]. The other included 505 patients from a Spanish registry [43]. Both studies reported exon 20 insertion prevalence within the range of values identified by this PLR, at $3.9 \%$ and $4.7 \%$ of all EGFR mutations, respectively $[42,43]$.

The prevalence estimates identified in this review and meta-analysis will be useful in calculations to estimate the upper and lower bounds of numbers of patients in European populations who would be eligible to receive emerging therapies that have specific activity against exon 20 insertion mutations, such as amivantamab or mobocertinib. Useful future work would include larger prospective cohort studies with longer follow-up periods to increase the likelihood of measuring precise results and validating the estimates of EGFR mutation prevalence; provide incidence data; and better clinically characterise the prognosis of patients with exon 20 insertion mutations. Regular updates to European analyses, along with an exploration of data in a wider range of countries, including real-world data from North America and Asia, would also be beneficial, particularly to capture key differences between regions.

Supplementary Information The online version contains supplementary material available at https://doi.org/10.1007/s11523-022-00868-z.

Acknowledgements The authors acknowledge Ruth Moulson, MPH from Costello Medical, London, for medical writing and editorial assistance based on the authors' input and direction. This study was sponsored by Janssen. Support for third-party writing assistance for this article, provided by Ruth Moulson, MPH, of Costello Medical, London was funded by Janssen in accordance with Good Publication Practice (GPP3) guidelines (http://www.ismpp.org/gpp3).

\section{Declarations}

Funding This work was funded by Janssen EMEA.

Conflict of interest SVS, NR, JS and BR and employees and shareholders of Janssen EMEA. MS has received personal fees from Amgen, AstraZeneca, Biontech, Bristol Myers Squibb, Boehringer Ingelheim, CureVac, Janssen-Cilag, Merck, MSD, Novartis, Pfizer, Roche, Sanofi-Aventis and Takeda; grants from AstraZeneca; and non-financial support from Bristol Myers Squibb, Pfizer and Takeda. AB, MM and DGL are employees of Costello Medical. CC has received personal fees from Amgen, AstraZeneca, Bayer, Boehringer Ingelheim, Bristol Myers Squib, Eli Lilly, GSK, Janssen, MSD, Novartis, Pfizer, Roche, Sanofi Aventis and Takeda. AG has received personal fees from Amgen, AstraZeneca, Boehringer Ingelheim, Bristol Myers Squibb, Eli Lilly, Janssen/Johnson \& Johnson, MSD, Novartis, Pfizer, Roche and Takeda; and research funding from AstraZeneca.
Ethics approval Not applicable

Consent to participate Not applicable.

Consent for publication Not applicable.

Availability of data and material Not applicable.

Code availability Not applicable.

Author contributions Substantial contributions to study conception and design: SVS, MM, AB, NR, JS, BR, DGL, CC, MS, AG; substantial contributions to analysis and interpretation of the data: SVS, MM, $\mathrm{AB}, \mathrm{NR}, \mathrm{JS}, \mathrm{BR}, \mathrm{DGL}, \mathrm{CC}, \mathrm{MS}, \mathrm{AG}$; drafting the article or revising it critically for important intellectual content: SVS, MM, AB, NR, JS, BR, DGL, CC, MS, AG; final approval of the version of the article to be published: SVS, MM, AB, NR, JS, BR, DGL, CC, MS, AG.

Open Access This article is licensed under a Creative Commons Attribution-NonCommercial 4.0 International License, which permits any non-commercial use, sharing, adaptation, distribution and reproduction in any medium or format, as long as you give appropriate credit to the original author(s) and the source, provide a link to the Creative Commons licence, and indicate if changes were made. The images or other third party material in this article are included in the article's Creative Commons licence, unless indicated otherwise in a credit line to the material. If material is not included in the article's Creative Commons licence and your intended use is not permitted by statutory regulation or exceeds the permitted use, you will need to obtain permission directly from the copyright holder. To view a copy of this licence, visit http://creativecommons.org/licenses/by-nc/4.0/.

\section{References}

1. Ferlay J, Colombet M, Soerjomataram I, Siegel R, Torre L, Jemal A. Global and regional estimates of the incidence and mortality for 38 cancers: GLOBOCAN 2018. World Health Organization, International Agency for Research on Cancer: Lyon, France. 2018.

2. Sung H, Ferlay J, Siegel RL, Laversanne M, Soerjomataram I, Jemal A, et al. Global cancer statistics 2020: GLOBOCAN estimates of incidence and mortality worldwide for 36 cancers in 185 countries. CA Cancer J Clin. 2021;71(3):209-49.

3. Eurostat. Cancer statistics - specific cancers 2020. https://ec. europa.eu/eurostat/statistics-explained/index.php?title=Cancer_ statistics_-_specific_cancers\#Lung_cancer. Accessed 21 May 2021.

4. National Cancer Institute Surveillance Epidemiology and End Results Program. Percent distribution and counts by histology among histologically confirmed cases, 2013-2017 both sexes by race 2017. https://seer.cancer.gov/archive/csr/1975_2017/results_ single/sect_15_table.28.pdf. Accessed 18 January 2022.

5. Zhu Q-G, Zhang S-M, Ding X-X, He B, Zhang H-Q. Driver genes in non-small cell lung cancer: characteristics, detection methods, and targeted therapies. Oncotarget. 2017;8(34):57680.

6. Pikor LA, Ramnarine VR, Lam S, Lam WL. Genetic alterations defining NSCLC subtypes and their therapeutic implications. Lung Cancer. 2013;82(2):179-89.

7. Bergethon K, Shaw AT, Ignatius Ou S-H, Katayama R, Lovly $\mathrm{CM}, \mathrm{McD}$ onald NT, et al. ROS1 rearrangements define a unique molecular class of lung cancers. J Clin Oncol. 2012;30(8):863-70.

8. Lynch TJBD, Sordella R, et al. Activating mutations in the epidermal growth factor receptor underlying responsiveness 
of non-small-cell lung cancer to gefitinib. N Engl J Med. 2004;350(21):2129-39.

9. Wee P, Wang Z. Epidermal growth factor receptor cell proliferation signaling pathways. Cancers (Basel). 2017;9(5):52.

10. Bethune G, Bethune D, Ridgway N, Xu Z. Epidermal growth factor receptor (EGFR) in lung cancer: an overview and update. $J$ Thorac Dis. 2010;2(1):48-51.

11. Zhang YL, Yuan JQ, Wang KF, Fu XH, Han XR, Threapleton $\mathrm{D}$, et al. The prevalence of EGFR mutation in patients with nonsmall cell lung cancer: a systematic review and meta-analysis. Oncotarget. 2016;7(48):78985-93.

12. Shigematsu H, Lin L, Takahashi T, Nomura M, Suzuki M, Wistuba II, et al. Clinical and biological features associated with epidermal growth factor receptor gene mutations in lung cancers. $\mathrm{J}$ Natl Cancer Inst. 2005;97(5):339-46.

13. Brindel A, Althakfi W, Barritault M, Watkin E, Maury J-M, Bringuier P-P, et al. Uncommon EGFR mutations in lung adenocarcinoma: features and response to tyrosine kinase inhibitors. J Thorac Dis. 2020;12(9):4643-50.

14. Vyse $\mathrm{S}$, Huang PH. Targeting EGFR exon 20 insertion mutations in non-small cell lung cancer. Signal Transduct Target Ther. 2019;4(1):1-10.

15. Yasuda H, Park E, Yun C-H, Sng NJ, Lucena-Araujo AR, Yeo W-L, et al. Structural, biochemical, and clinical characterization of epidermal growth factor receptor (EGFR) exon 20 insertion mutations in lung cancer. Sci Transl Med. 2013;5(216):216ra177.

16. Wang F, Li C, Wu Q, Lu H. EGFR exon 20 insertion mutations in non-small cell lung cancer. Transl Cancer Res. 2020;9(4):2982-91.

17. Ellison G, Zhu G, Moulis A, Dearden S, Speake G, McCormack R. EGFR mutation testing in lung cancer: a review of available methods and their use for analysis of tumour tissue and cytology samples. J Clin Pathol. 2013;66(2):79-89.

18. Illumina. Advantages of next-generation sequencing vs. qPCR 2021. https://www.illumina.com/science/technology/next-gener ation-sequencing/ngs-vs-qpcr.html. Accessed 29 July 2021.

19. Sousa AC, Silveira C, Janeiro A, Malveiro S, Oliveira AR, Felizardo M, et al. Detection of rare and novel EGFR mutations in NSCLC patients: implications for treatment-decision. Lung Cancer. 2020;139:35-40.

20. Xie T, Zou Z, Liu C, Zhu Y, Xu Z, Wang L, et al. Front-line therapy in EGFR exon 19 deletion and 21 Leu858Arg mutations in advanced non-small cell lung cancer: a network meta-analysis. Evid Based Complem Altern Med. 2021;2021:1-15.

21. Yasuda H, Ichihara E, Sakakibara-Konishi J, Zenke Y, Takeuchi S, Morise M, et al. A phase I/II study of osimertinib in EGFR exon 20 insertion mutation-positive non-small cell lung cancer. Lung Cancer. 2021;162:140-6.

22. Le X, Goldman JW, Clarke JM, Tchekmedyian N, Piotrowska Z, $\mathrm{Chu} \mathrm{D}$, et al. Poziotinib shows activity and durability of responses in subgroups of previously treated EGFR exon 20 NSCLC patients. J Clin Oncol. 2020;38(15_suppl):9514.

23. Burnett H, Emich H, Carroll C, Stapleton N, Mahadevia P, Li T. Epidemiological and clinical burden of EGFR Exon 20 insertion in advanced non-small cell lung cancer: a systematic literature review. PLoS ONE. 2021;16(3):e0247620.

24. Nicolas Girard LB, Minchom A, Ignatius Ou S-H, Gadgeel S, Trigo J, Viteri S, Li G, Mahadevia P, Londhe A, Backenroth D, $\mathrm{Li} \mathrm{T}$, Bauml T, editor. Comparative clinical outcomes for patients with NSCLC harbouring EGFR exon 20 insertion mutations and common EGFR mutations. In: 2020 World conference on lung cancer; 2020; Singapore 2020

25. U.S Food \& Drug Administration. FDA grants accelerated approval to amivantamab-vmjw for metastatic non-small cell lung cancer 2021. https://www.fda.gov/drugs/resources-infor mation-approved-drugs/fda-grants-accelerated-approval-amiva ntamab-vmjw-metastatic-non-small-cell-lung-cancer. Accessed 18 January 2022.

26. European Medicines Agency. Rybrevant Opinion 2021. https:// www.ema.europa.eu/en/medicines/human/summaries-opinion/ rybrevant. Accessed 18 January 2022.

27. U.S Food \& Drug Administration. FDA grants accelerated approval to mobocertinib for metastatic non-small cell lung cancer with EGFR exon 20 insertion mutations 2021. https://www.fda. gov/drugs/resources-information-approved-drugs/fda-grants-accel erated-approval-mobocertinib-metastatic-non-small-cell-lungcancer-egfr-exon-20. Accessed 18 January 2022.

28. Shea BJ, Reeves BC, Wells G, Thuku M, Hamel C, Moran J, et al. AMSTAR 2: a critical appraisal tool for systematic reviews that include randomised or non-randomised studies of healthcare interventions, or both. BMJ. 2017;358:j4008.

29. Joanna Briggs Institute. The Joanna Briggs Institute Critical Appraisal tools for us in JBI systematic reviews checklist for prevalence studies. 2017. University of Adelaide, Australia. https://jbi. global/sites/default/files/2019-05/JBI_Critical_Appraisal-Check list_for_Diagnostic_Test_Accuracy_Studies2017_0.pdf. Accessed 29 July 2020.

30. Team RC. R: a language and environment for statistical computing. 2013.

31. Balduzzi S, Rücker G, Schwarzer G. How to perform a metaanalysis with R: a practical tutorial. Evid Based Ment Health. 2019;22(4):153-60.

32. Beau-Faller M, Prim N, Ruppert AM, Nanni-Metellus I, Lacave R, Lacroix L, et al. Rare EGFR exon 18 and exon 20 mutations in non-small-cell lung cancer on 10117 patients: a multicentre observational study by the French ERMETIC-IFCT network. Ann Oncol. 2014;25(1):126-31.

33. Mansuet-Lupo A, Zouiti F, Alifano M, Tallet A, Charpentier M-C, Ducruit $\mathrm{V}$, et al. Intratumoral distribution of EGFR mutations and copy number in metastatic lung cancer, what impact on the initial molecular diagnosis? J Trans Med. 2014;12(1):131.

34. Mansuet-Lupo A, Bobbio A, Blons H, Becht E, Ouakrim H, Didelot A, et al. The new histologic classification of lung primary adenocarcinoma subtypes is a reliable prognostic marker and identifies tumors with different mutation status: the experience of a French cohort. Chest. 2014;146(3):633-43.

35. Locatelli-Sanchez M, Couraud S, Arpin D, Riou R, Bringuier PP, Souquet PJ. Routine EGFR molecular analysis in non-smallcell lung cancer patients is feasible: exons 18-21 sequencing results of 753 patients and subsequent clinical outcomes. Lung. 2013;191(5):491-9.

36. Stella GM, Scabini R, Inghilleri S, Cemmi F, Corso S, Pozzi E, et al. EGFR and KRAS mutational profiling in fresh nonsmall cell lung cancer (NSCLC) cells. J Cancer Res Clin Oncol. 2013;139(8):1327-35.

37. Moore DA, Balbi K, Ingham A, Arkenau HT, Bennett P. Analysis of a large cohort of non-small cell lung cancers submitted for somatic variant analysis demonstrates that targeted next-generation sequencing is fit for purpose as a molecular diagnostic assay in routine practice. J Clin Pathol. 2018;71(11):1001-6.

38. Evans M, O'Sullivan B, Smith M, Hughes F, Mullis T, Trim N, et al. Large-scale EGFR mutation testing in clinical practice: analysis of a series of 18,920 non-small cell lung cancer cases. Pathol Oncol Res. 2019;25(4):1401-9.

39. Kerner GS, Schuuring E, Sietsma J, Hiltermann TJ, Pieterman RM, de Leede GP, et al. Common and rare EGFR and KRAS mutations in a Dutch non-small-cell lung cancer population and their clinical outcome. PLoS ONE. 2013;8(7):e70346.

40. Sandelin M, Berglund A, Sundstrom M, Micke P, Ekman S, Bergqvist M, et al. Patients with non-small cell lung cancer analyzed for EGFR: adherence to guidelines, prevalence and outcome. Anticancer Res. 2015;35(7):3979-86. 
41. Vazquez S, Casal J, Afonso FJA, Firvida JL, Santome L, Baron $\mathrm{F}$, et al. EGFR testing and clinical management of advanced NSCLC: A galician lung cancer group study (GGCP 048-10). Cancer Manag. 2016;8:11-20.

42. Chouaid C, Filleron T, Debieuvre D, Pérol M, Girard N, Dansin E, et al. A real-world study of patients with advanced non-squamous non-small cell lung cancer with EGFR exon 20 insertion: clinical characteristics and outcomes. Target Oncol. 2021;16(6):801-11.
43. Gutiérrez L, Royuela A, Carcereny E, López-Castro R, RodríguezAbreu D, Massuti B, et al. Prognostic model of long-term advanced stage (IIIB-IV) EGFR mutated non-small cell lung cancer (NSCLC) survivors using real-life data. BMC Cancer. 2021;21(1):1-11. 